\title{
Researching of Intelligence Test Paper Generation Software based on Improved Genetic Algorithm in Java Program Design Teaching
}

\author{
Qiufang Ma ${ }^{1, a}$, Hao Pan 2, b \\ ${ }^{1}$ College of International Electronic Commerce, Qingdao Huanghai University, Qingdao 266427, \\ China; \\ ${ }^{2}$ College of Information \& Control Engineering, China University of Petroleum, Qingdao 266580, China. \\ amfant2005@163.com, briverhp@126.com
}

Keywords: java program design, genetic algorithm, intelligent test paper generation.

\begin{abstract}
Editing test papers is a very heavy mental labor. Aiming to improve the teaching effect of Java Program Design and realize the separation of teaching and test, the intelligent test paper generation software based on improved genetic algorithm is designed. The software provides three generating methods based on guaranteeing the reasonable distribution of knowledge points. The teaching practice results demonstrate that the application of the software can improve teaching quality effectively, achieve standardization of test and promote the development of teachers.
\end{abstract}

\section{Introduction}

Examination is an important part in the process of teaching. It is the important method to learn about how well the students have mastered the knowledge [1]. Traditional test mainly uses the paper do a form. Teachers take a lot of time and energy to collect paper, compilation, sorting, and analysis of test paper in the teaching process. It is low efficiency.

Java is an object-oriented programming language, it have some advantaged features such as cross-platform, distributed, high safety, robustness, and multi-threading [2]. It was favorable by programmers around the world since it launched. Java has been successfully applied in consumer electronics, smart phones, desktop applications, enterprise applications, and other fields. It was not only the most popular and widespread program language in IT and education world. Now, the most college and university all over the world have set up this course. Java has been the important branch of university program design teaching and major compulsory course of computer science and technology in our college. This course aims to enable students to master the core concept and programming methods in Java technology and training students to master the object-oriented thought and program design method. The students can master the language that is very important in scientific research and market application. After completing this course of study, the students can use java technology and object-oriented thought to solve problems in real life. But this course has scattered and widely knowledge, so it is hard to learning.

Currently, Computer assisted Instruction(CAI) has become an important part of modern higher education [3], and intelligence test paper generation is the base of Computer -based Testing(CBT). The intelligence test paper generation is choosing questions by the computer automatically from the test database. We can conform to the test paper that meet demand, such as specific score, total time, test question type, test question quantity, knowledge points and so on[4].

Compared with the manual editing test papers, the intelligence test paper generation has the following advantages:

The intelligence test paper generation can according to the requirement of the teacher generate the test paper that meet a set of condition demands, greatly shorten the time and reduce the burden of the teacher.

2. Each question in the test database is according to the experience of the investigation of teacher and student, so the test paper generated from the intelligence test paper generation software is accuracy and scientific, can measure students true ability. 
3. The intelligence test paper generation can be impartial, can promote the improvement of teachers teaching level. It avoiding teacher divulge the information before test.

4. The intelligence test paper generation can improve the level of teaching management, make the test work more standardized, more scientific, and increases the impartiality and fairness of the test.

We classified the knowledge points of java program design and developed the intelligence test paper generation software based on improved genetic algorithm. The intelligence test paper generation software established a reasonable test database that contains four different test question type such as choice questions, completion, true or false questions, programming questions. We applied this software in the teaching of java program design, and realize the separation of teaching and testing. The teachers can be extricated from the repeated editing test papers. This software not only improved the work efficiency, but also accelerate the progress of the test digitization and informatization.

\section{The Intelligence Test Paper Generation Software Based on Improved Genetic Algorithm in JAVA Program Design}

The main problem in the researching of intelligence test paper generation is how to ensure the randomness, scientific and reasonable of the generated test paper [5]. As a key technology to realize the intelligence test paper generation, high efficiency, scientific generation algorithm is one of the hot research topic in the field of computer assisted instruction. As a mimic natural biological evolution process of the intelligent algorithm, genetic algorithm has parallelism, global optimization and fast convergence speed and so on characteristics, has been widely used in intelligence test paper generation. But simple genetic algorithm has the problem of poor local search ability and premature convergence, so it need to improve in actual application. This paper improves the genetic operators, proposes a segmentation more hybrid strategy is directed against hybrid operator, proposes a segmentation more mutation strategy is directed against mutation operator. This strategy can effectively avoid knowledge conflicts in the process of generate test paper, and use the large ratio of hybridization and mutation to keep the diversity of population, avoiding the quickly narrow the search space, improves the global optimization performance, speed up the whole algorithm to approach the global optimal value of the speed. At the same time, in the process of genetic use the optimal preservation strategy select offspring and ensure the good parent individuals into the children.

The software based on Matlab and use GUI made a concise and efficient interface, ultimately generated executable file that can run without Matlab. Combining the syllabus of JAVA program design, course group divide the course into 28 knowledge points, such as JAVA operating mechanism, JAVA operators and expressions, class member variables and methods of access, the main classes in the Java. lang package, and Java exception handling mechanism. On this basis, the course group established the reasonable test database that contains multiple choice questions, fills up the topic, true or false, programming problem and so on four different question types, and improved the intelligence test paper generation software based on improved genetic algorithm in java program design. The intelligence test paper generation software hardware environment: CPU: more than $1.5 \mathrm{GHz}$; Memory: $512 \mathrm{MB}$ or more; Hard disk: more than 50G; Software environment, the operating system: Windows 2003 / XP / 2003 / Vista/Windows 7 / doing/Win10.The intelligence test paper generation software based on improved genetic algorithm in java program design including the welcome interface, the main interface, software introduction interface, query knowledge points interface, modify test questions attributes interface, modify correlation coefficient of knowledge points interface, set related parameters of the test paper interface and the interface of intelligent test paper generation based on improved genetic algorithm. The detailed description of several important interface of this software will be given in the following.

\subsection{The Main Interface}

As shown in figure 1, the main interface consists of six parts: the introduction of the software, query knowledge points of course section, modify the test paper attributes section, modify correlation coefficient of knowledge points section, set related parameters of the test paper section, and the test 
paper generation section. Click the "software introduction" button, you can enter software introduction interface. Click the "query knowledge points" button, can enter query knowledge points interface, as shown in figure 2 . Click the "modify test attributes" button, you can enter into the interface of modify test paper attributes, as shown in figure 3. Click the "modify correlation coefficient" button, you can enter the interface of modify correlation coefficient of knowledge points, as shown in figure 7. Click the "set related parameters" button, you can enter the interface of set the test paper related parameters. Click the "test paper generation" button, can enter the interface of intelligent test paper generation based on improved genetic algorithm. Click "quit" button, then shut down the interface.

The Intelligence Test Paper Generation Software

Based on Improved Genetic Algorithm in JAVA Program Design

Software Introduction

Modify Test Attributes

Set Related Parameters
Query Knowledge Point

Modify Correlation Coefficient

Test Paper Generation
QUIT

Fig. 1 Main interface

\section{Query Knowledge Points}
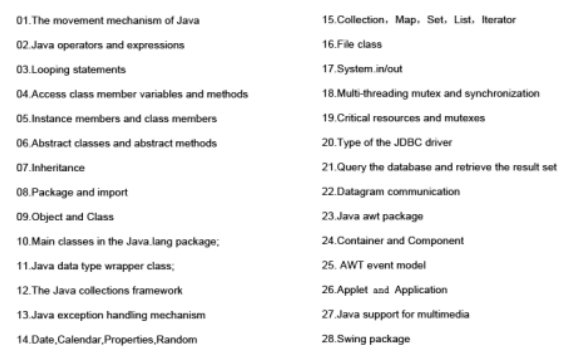

OK

Fig. 2 Query knowledge point interface

\subsection{The Modify Test Paper Attributes Interface}

The interface of figure 3 consists of three parts: the parameter input part (text paper number), paper attributes display section and modification section. User input test paper code , click on the "query" button, it can show test paper attributes, including the type of questions (multiple choice, completion, true or false, programming problem), test difficulty coefficient, time consuming (minutes), test score, etc., as shown in figure 4. Click "quit" button, then shut down the interface.

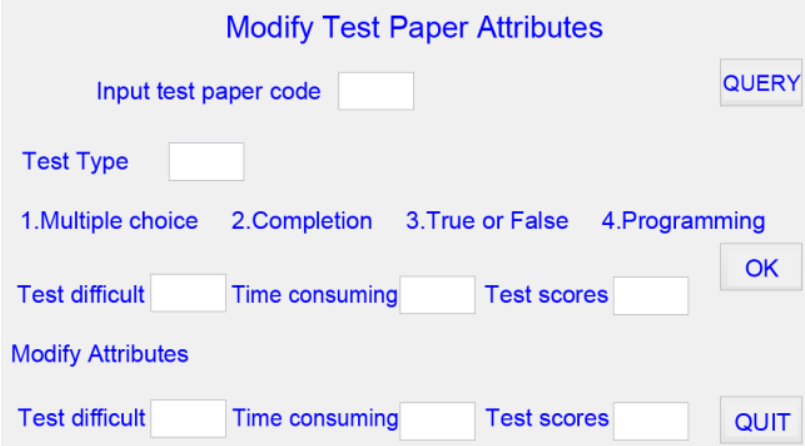

Fig. 3 Modify test attributes interface (1)

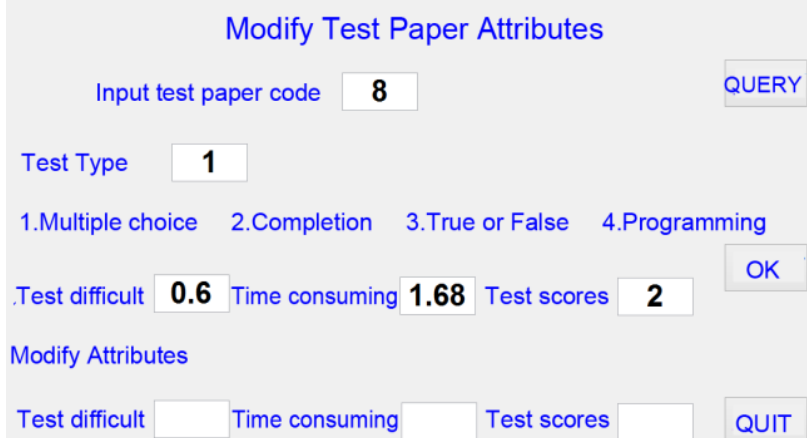

Fig. 4 Modify test attributes interface (2)

Questions of attributes preservation to the software, as shown in figure 5. Click on the "query" button again, can show the modified paper attributes, as shown in figure 6 .

\section{Modify Test Paper Attributes}

Input test paper code 8

\begin{tabular}{l|l} 
Test Type & 1
\end{tabular}

1.Multiple choice 2.Completion 3.True or False 4.Programming

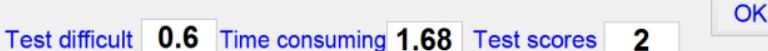

Modify Attributes

\begin{tabular}{l|l|l|l|} 
Test difficult & $\mathbf{0 . 5 2}$ Time consuming $\mathbf{1 . 5 0}$ Test scores & $\mathbf{2}$ & QUIT
\end{tabular}

Fig. 5 Modify test attributes interface (3)
Modify Test Paper Attributes

Input test paper code 8

QUERY

Test Type 1

1.Multiple choice 2.Completion 3.True or False 4.Programming

Test difficult $\mathbf{0 . 5 2}$ Time consuming $\mathbf{1 . 5}$ Test scores 2

OK

Modify Attributes

Test difficult $\quad$ Time consuming $\quad$ Test scores

QUIT

Fig. 6 Modify test attributes interface (4)

\subsection{The Modify Correlation Coefficient of Knowledge Points Interface}

The interface of figure 7 consists of three parts: the knowledge correlation coefficient shows section, the parameter input section (number), the modify correlation coefficient of knowledge points . Click on the "query" button, it can show knowledge correlation coefficient of this course. In this software, we set the default initial value of different knowledge is 0.5 , the same knowledge is 1 . The user can 
according to actual situation, combined with their own experience, modify the corresponding knowledge correlation coefficient after input the knowledge point number, as shown in figure 8, 9. Click "ok" button, the modified correlation coefficient of knowledge can be saves in this software. Click on the "query" button again, can show the modified knowledge correlation coefficient. Click "quit" button, then shut down the interface.

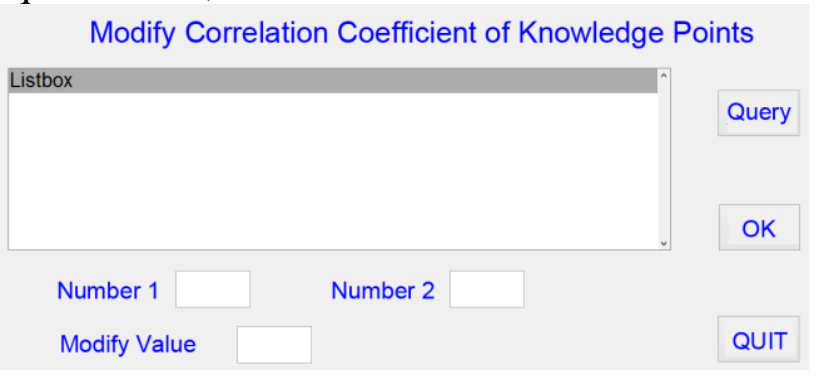

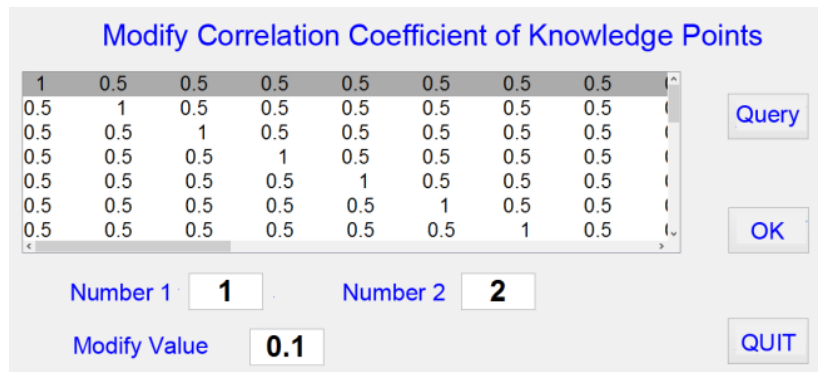

Fig. 7 Modify correlation coefficient interface (1) Fig. 8 Modify correlation coefficient interface (2)

\section{Summary}

We applied intelligence test paper generation software in java program design to the students who major in computer science and technology. In the process of teaching, the classroom atmosphere is very active, students' learning initiative and enthusiasm is increased greatly. The test scores of this course according with normal distribution, passing rate was $87 \%$.On the whole, the effect is very ideal. This software is to improve students' learning interest and teaching quality, to achieve the test standardization, is helpful to improve the level of teaching teachers' teaching. This software can also be combined with Computer assistant instruction software of java programming design, to improve the quality of classroom teaching.

\section{Acknowledgements}

Fund Project: Shandong province education science five-year planning topics for 2015: Modern information technology and the android development of curriculum integration research (Project Number: YBJ15008)

\section{References}

[1]. Yali Zhang, Minggui Qu, Qingxiang Yang, Chao Zhang. The Analysis of Higher School Curriculum Examination Reform. Education and teaching research. Vol. 24 (2011) No. 4, p.58-59.

[2]. Junhai Yong. Java Programming Design. Tsinghua university press, 2007, p.2-3.

[3]. Ya Huang, Xing Yan. Introduction to Computer Aided Teaching Platform Construction. China Electric Power Education. 2006(3), p.43-344.

[4]. Rong He, Shuang Chen. Research and Design on On-line Generating Paper Algorithm. Computer Engineering and Design. Vol. 32 (2011) No. 6, p.2183-2184.

[5]. Houchong Wan. The Improved Adaptive Genetic Algorithm in the Application of the Intelligence Test Paper Generation. Fujian Computer. 2010(1), p.119-120.

[6]. Xiangyang Niu. Genetic algorithm Coupled with Other Intelligent Algorithm and its Application Research. Science and Technology Innovation Herald. (2010) No. 35, p.212. 